\title{
Galaxy Evolution through spectral fitting tools: A comparative study between STECKMAP and FADO
}

\section{Ciro Pappalardo(iD, Polychronis Papaderos, Jean Michel Gomes, Leandro Cardoso, Ana Afonso, Israel Matute and Stergios Amarantidis}

\author{
Instituto de Astrofísica e Ciências do Espaço, Portugal
} email: ciro@oal.ul.pt

\begin{abstract}
Spectral analysis is nowadays a widely used tool to investigate the evolution of galaxies. Assessing the reliability of this approach is crucia, motivating a through analysis. In this poster, a comparative study between two widely tools, FADO and STECKMAP, is performed, focusing on the discrepancies between the different approaches. Both codes use different methods to extract the best fit, allowing the possibility to disentangle possible biases introduced in the analysis. Our analysis showed that where nebular emission is not negligible, the results obtained with methods taking into account such a component are more reliable, and this can be very important when moving at higher redshift, where stellar populations are younger. In particular, this is true for starburst systems, where a huge amount of stars are forming almost at the same epoch. This is an important aspect to take into account the future survey, as JWST for example, which will provide the community with medium resolution spectra of galaxies at redshift 3-4 and even higher.
\end{abstract}

Keywords. Spectral fitting tools, Galaxy Evolution, Nebular emission

A fundamental tool to investigate the evolution of galaxies is the analysis of the spectra obtained through the decomposition of the light received from these objects. Spectra contain crucial information about the stellar and gas emission, chemistry, and kinematics. However, to apply such an approach we must be absolutely confident about the reliability of the results. With the increase of technical capabilities, and with all a new generation of instruments, different spectral analysis tools have been proposed to the scientific community in order to extract such parameters, e.g. STECKMAP, FireFly, FADO, and VESPA Ocvirk et al. (2006); Wilkinson et al. (2017); Gomes \& Papaderos (2017); Tojeiro et al. (2009). But still, many biases and inconsistencies in the results are present, with scientists finding different results using different tools.

In this poster, a comparative study for some of these spectral fitting tools is performed, focusing on the discrepancies between the different approaches. Using the evolutionary stellar population code REBETIKO, a set of different mock spectra, with different SNR, and star formation histories are produced (Fig. 1). These spectra are analyzed with two different tools: FADO and STECKMAP (Fig. 2). Both codes use different methods to extract the best fit, allowing the possibility to disentangle possible biases introduced in the analysis. STECKMAP reproduces quite well the spectra and the star formation history of the mock data, even if for some particular model a secondary peak appears. This double peak is due to a bad spectral coverage within the time bin of the secondary peak since the proposed spectral synthesis population basis does not have a sufficient number of spectra covering that specific time bin. Moreover, we note that the typical double 


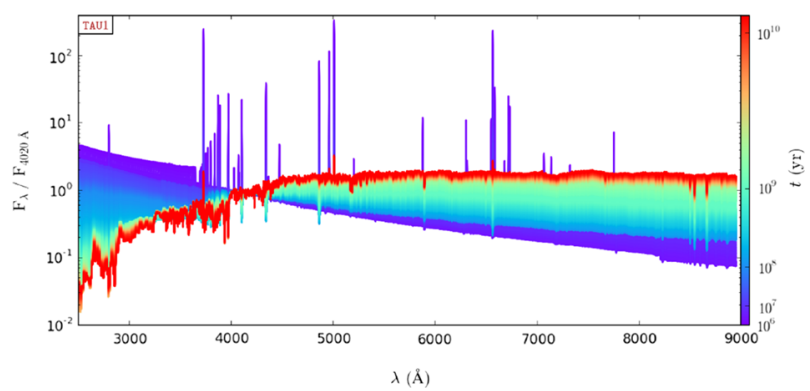

Figure 1. Mock spectra produced using rebetiko code, considering an exponentially decreasing star formation history ( $\tau=1 \mathrm{Gyr})$, solar metallicity, and no extinction, color coded according to the age (color bar on the right). Spectra are normalized at $4020 \AA$.
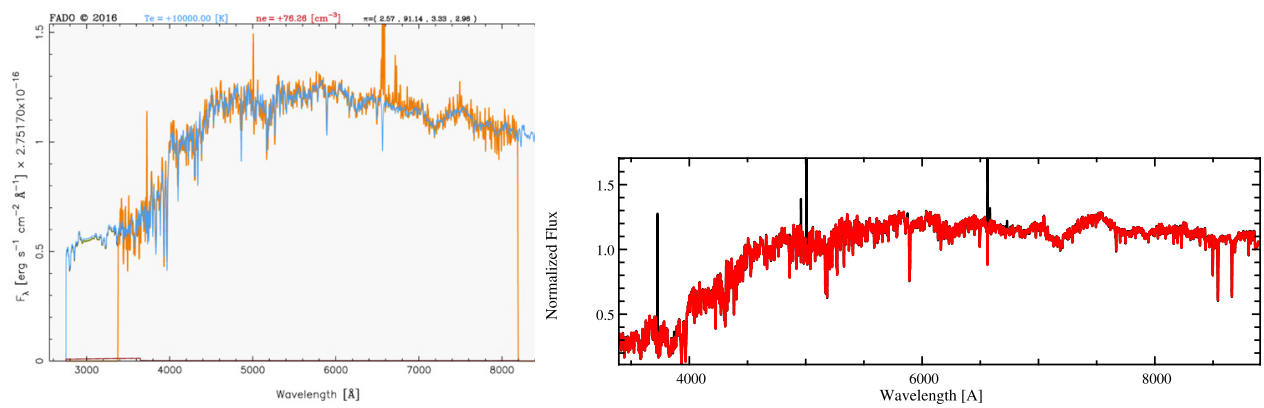

Figure 2. Examples of spectral fit for FADO (left panel) and STECKMAP (right panel).

peak is on average shifted towards the younger or the older time bin. This introduces an interesting feature because the oldest time bin corresponds to a stellar population with high weight in terms of total mass and low weight in terms of total light, while the young one has a low weight in terms of mass but high weight in terms of light. This trend can have different origins: for example, these secondary peaks are particularly evident when considering stellar populations at an epoch where the nebular emission is still relevant. In those cases, FADO reproduces better the trend for both nebular and stellar continuum emission, confirming that at these stages of star formation the nebular emission must be properly taken into account. Where nebular emission is not negligible, the results obtained with methods taking into account such a component are more reliable, and this can be very important when moving at higher redshift, where the stellar populations are young. In particular, this is true for starburst systems, where a huge amount of stars are forming almost at the same epoch. This is an important aspect to take into account the future survey, as JWST for example, which will provide the community with medium resolution spectra of galaxies at redshift 3-4 and even higher. For these objects, it is important to quantify these effects, as the emission due to the ionized gas can be very high compared to the emission of the stellar continuum.

\section{References}

Gomes, J. M. \& Papaderos, P. 2017, A\&A A, 603, A63

Ocvirk, P., Pichon, C., Lançon, A., \& Thiébaut, E. 2006, MNRAS, 365, 74

Wilkinson, D. M., Maraston, C., Goddard, D., Thomas, D., \& Parikh, T. 2017, MNRAS, 472,4297

Tojeiro, R., Wilkins, S., Heavens, A. F., Panter, B., \& Jimenez, R. 2009, ApJS, 185, 1 